\title{
Descrição da larva de Cosmariomyia argyrosticta Kertész e do pupário de Dactylodeictes lopesi Lindner (Diptera, Stratiomyidae)
}

\author{
Roberto de Xerez ${ }^{1,3}$ \\ José Roberto Pujol-Luz 2 \\ Gustavo Glória Viana ${ }^{1}$
}

\begin{abstract}
Description of the larva of Cosmariomyia argyrosticta Kertész and the puparium of Dactylodeictes lopesi Lindner (Diptera, Stratiomyidae). The larva of Cosmariomyia argyrosticta Kertész, 1914 and the puparium of Dactylodeictes lopesi Lindner, 1964 are described for the first time, based on four larvae and 10 puparia and one puparium respectively. Larvae were collected under the bark of fallen trees in an area near of tropical rain forest at Iguaba Grande $\left(22^{\circ} 50^{\prime} 21^{\prime \prime} \mathrm{S}, 42^{\circ} 13^{\prime} 44^{\prime \prime} \mathrm{W}\right.$, $18 \mathrm{~m}$ ) and Búzios ( $22^{\circ} 44^{\prime} 49^{\prime \prime} \mathrm{S}, 41^{\circ} 52^{\prime} 54^{\prime \prime} \mathrm{W}, 3 \mathrm{~m}$ ), State of Rio de Janeiro, Brazil. Some biological notes are also presented. The larvae of Cosmariomyia argyrosticta and Dactylodeictes lopesi are compared with Chalcidomorphina aurata Enderlein, 1914 and Vittiger schnusei Kertész, 1909.

KEY WORDS. Stratiomyidae, Pachygastrinae, Cosmariomyia argyrosticta, Dactylodeictes lopesi, soldier flies
\end{abstract}

Os Stratiomyidae são braquíceros da Infra-ordem Stratiomyomorpha. A classificação mais recente do grupo foi apresentada por WOODLEY (1989) compreendendo duas famílias: Xylomyidae e Stratiomyidae. De acordo com WoODLEY $(1989,1995)$, os Stratiomyidae são reconhecidos pelos seguintes caracteres (1 a 4 = Stratiomyomorpha; 5 a 8 = autapomorfias de Stratiomyidae): (1) pupário formado do tegumento do último estágio larval, (2) cutícula larval impregnada de carbonato de cálcio, (3) perda do esporão das pernas anteriores, (4) veia costal abreviada, (5) perda do esporão da tíbia média, (6) cercos da fêmea separados pelo tergito 10, (7) veias radiais agrupadas para frente da margem costal da asa e (8) célula discal reduzida em tamanho.

Segundo WoOdLEY (2001), doze subfamílias ocorrem na região Neotropical com 987 espécies descritas: Parhadrestriinae, Chiromyzinae, Beridinae, Sarginae, Raphiocerinae, Clitellariinae, Chrysochlorininae, Hermetiinae, Stratiomyinae, Antissinae, Nemotelinae e Pachygastrinae.

Segundo JAMES (1981), larvas de Pachygastrinae podem ser reconhecidas pela seguinte combinação de caracteres: (1) segmento terminal do abdômen sem cerdas hidrófobas, (2) cerdas dorsais não circundadas por pequenas cerdas, (3) lábio

1) Departamento de Biologia Animal, Instituto de Biologia, Universidade Federal Rural do Rio de Janeiro. Rodovia BR 465, Km 07, 23890-000 Seropédica, Rio de Janeiro, Brasil.

2) Departamento de Zoologia, Instituto de Biologia, Universidade de Brasília. 70910-900 Brasilia, Distrito Federal, Brasil. E-mail: jrpujol@unb.br

3) E-mail: rdexerez@ufrrj.br 
pouco desenvolvido ou ausente, (4) complexo mandíbulo-maxilar do aparelho bucal em parte fortemente esclerotizado, (5) ausência de ganchos nos esternitos abdominais 5-7, (6) ausência de espinhos na abertura anal.

Das 136 espécies de Pachygastrinae ocorrentes na região neotropical (WoODLEY 2001), são conhecidas e descritas as larvas de somente 4 espécies (Eupachygaster alexanderi (James, 1967), Zabrachia stoichoides James, 1965, Chalcidomorphina aurata Enderlein, 1914 e Vittiger schnusei Kertész, 1909).

BLANCHARD (1923) publicou a descrição da larva de E. alexanderi (JAMES 1967b) mencionando a coloração da larva (amarelada), a forma do corpo (achatada) e a presença de várias cerdas e informou, também, que a larva era saprófita e foi encontrada em talos putrefatos de Opuntia sulphurea G. Don, 1830 (Cactaceae). Mencionou, ainda, que seu desenvolvimento deveria ser muito lento, pois larvas coletadas em dezembro demoraram cerca de três meses para completar seu desenvolvimento.

JAMES (1965) descreveu a larva de Z. stoichodes. Em seu trabalho, o autor constatou que as cerdas medianas mesotorácicas eram maiores do que as mais internas, a cerda mais externa do metatórax era muito pequena e a mais interna maior que a mediana. Citou a coloração do pupário, marrom amarelada na região anterior e marrom na parte posterior dos segmentos. Apresentou um desenho mostrando os três últimos segmentos abdominais em vista dorsal e ventral.

A larva de C. aurata foi descrita por Pujol-Luz \& Xerez (1999) com base em duas larvas e quatro pupários coletados sob casca de árvores na Ilha da Marambaia, Mangaratiba, Rio de Janeiro, em 1998. A larva e o pupário foram descritos com detalhes de comprimento, forma e coloração geral, quetotaxia da cabeça, tórax e abdômen, informando quantidade, localização e forma das cerdas, e caracterização da mancha ventral do sexto segmento abdominal. Foram também fornecidos detalhes sobre a biologia das larvas observadas no campo e no laboratório.

XEREZ \& PUJOL-LUZ (2001) descreveram a larva de $V$. schnusei com base em duas larvas e dois pupários coletados sob casca de árvores na Ilha da Marambaia, Mangaratiba, Rio de Janeiro, em 1998. A descrição foi feita com os mesmos detalhes que em $C$. aurata. Os autores apresentaram uma comparação com a larva de $C$. aurata com base em características morfológicas e de quetotaxia.

Cosmariomyia Kertész, 1914, com três espécies, distribui-se na região Neotropical de El Salvador até a Argentina: Cosmariomyia albarista James, 1980 (El Salvador) (in: JAMES et al. 1980); Cosmariomyia argyrosticta Kertész, 1914 (Brasil; Argentina; Costa Rica e El Salvador) e Cosmariomyia pallidipenis (Williston, 1901) (México) (WoOdLEy 2001).

Dactylodeictes Kertész, 1914, com quatro espécies, tem a seguinte distribuição: Dactylodeictes amazonicus Kertész, 1914 (Brasil); D. brevifacies James, 1974 (Peru); D. lopesi Lindner, 1964 (México, Panamá, Trinidad e Brasil) e D. medius James, 1974 (Equador e Peru) (WoOdLEy 2001).

Neste trabalho descreve-se a larva de $C$. argyrosticta e o pupário de $D$. lopesi e compara-se com as larvas de C. aurata e V. schnusei. 


\section{MATERIAL E MÉTODOS}

As larvas de C. argyrosticta foram coletadas em Iguaba Grande (22 50 ' $21^{\prime \prime} \mathrm{S}$, $42^{\circ} 13^{\prime} 44^{\prime \prime} \mathrm{W}, 18 \mathrm{~m}$ ) e as de D. lopesi em Búzios (2244'49'’ $, 41^{\circ} 52^{\prime} 54^{\prime \prime} \mathrm{W}, 3 \mathrm{~m}$ ), Rio de Janeiro, Brasil, em ambos os casos sob a casca de árvores caídas em estágio inicial de decomposição. Um total de 97 larvas foi coletado (94 de C. argyrosticta e três de $D$. lopesi), transportadas para o laboratório e acondicionadas em placas de Petri ( $6 \mathrm{~cm}$ de diâmetro), tendo cada placa no máximo quatro larvas, com substrato onde foram coletadas (fungos, madeira em decomposição e exudato). Diariamente foram feitas observações para verificar se houve ecdise ou emergência e adicionadas algumas gotas de água quando o substrato se apresentava seco. Após a emergência, transferia-se o adulto para um tubo de vidro. Vinte e quatro horas após a emergência, os adultos eram fixados, utilizando-se éter sulfúrico ou clorofórmio; procedia-se à montagem do exemplar e os pupários eram colocados em microtubos plásticos de $1,4 \mathrm{~cm}$ de comprimento, contendo álcool $70 \%$ e glicerina (2:1) e anexado ao alfinete de montagem do adulto. Quando existiam várias larvas da mesma coleta e havia a constatação da existência de pupas, eram fixados pelo menos dois exemplares, mantidos em pequenos frascos etiquetados, hermeticamente fechados, contendo álcool $70 \%$.

Todo o material coletado foi depositado na Coleção Entomológica Costa Lima da Universidade Federal Rural do Rio de Janeiro.

\section{RESULTADOS}

\section{Cosmariomyia Kertész, 1914}

Cosmariomyia Kertész, 1914: 552. Espécie tipo: Cosmariomyia argyrosticta Kertész (des. orig.).

\section{Cosmariomyia argyrosticta Kertész, 1914}

\section{Figs 1-6}

Distribuição. Neotropical: Argentina (Salta, Tucumán); Brasil: Rio de Janeiro (nova ocorrência), São Paulo e Santa Catarina; Costa Rica; El Salvador.

Larva. Comprimento 4,9 a 6,4 $\mathrm{mm}$, achatada dorsoventralmente, margem lateral dos segmentos do corpo arqueadas. Cutícula com a usual aparência de mosaico, algumas células formando manchas características na região dorsolateral dos segmentos abdominais. Padrão cromático marrom-amarelado.

Cabeça. Achatada dorsoventralmente (Fig.1); comprimento da cabeça maior do que a largura (Fig. 2); complexo mandíbulo-maxila bem desenvolvido, escovas cilíndricas quase tão longas quanto o labro em vista dorsal; labro triangular. Antena curta, surgindo na parte anterior da cabeça. Olhos proeminentes, arredondados, aparecendo na parte posterior da cabeça. Quetotaxia: dois pares de cerdas clipeofrontais, um par de cerdas dorsolaterais inseridas acima dos olhos, um par de cerdas laterais inseridas abaixo dos olhos, três pares de cerdas ventrolaterais e três pares de cerdas ventrais em forma de espinho (Figs 1 e 2).

Tórax. Primeiro segmento torácico menor do que os outros. Espiráculo proeminente com um par de cerdas dorsolaterais e um par de cerdas ventrolaterais (Fig. 1). 

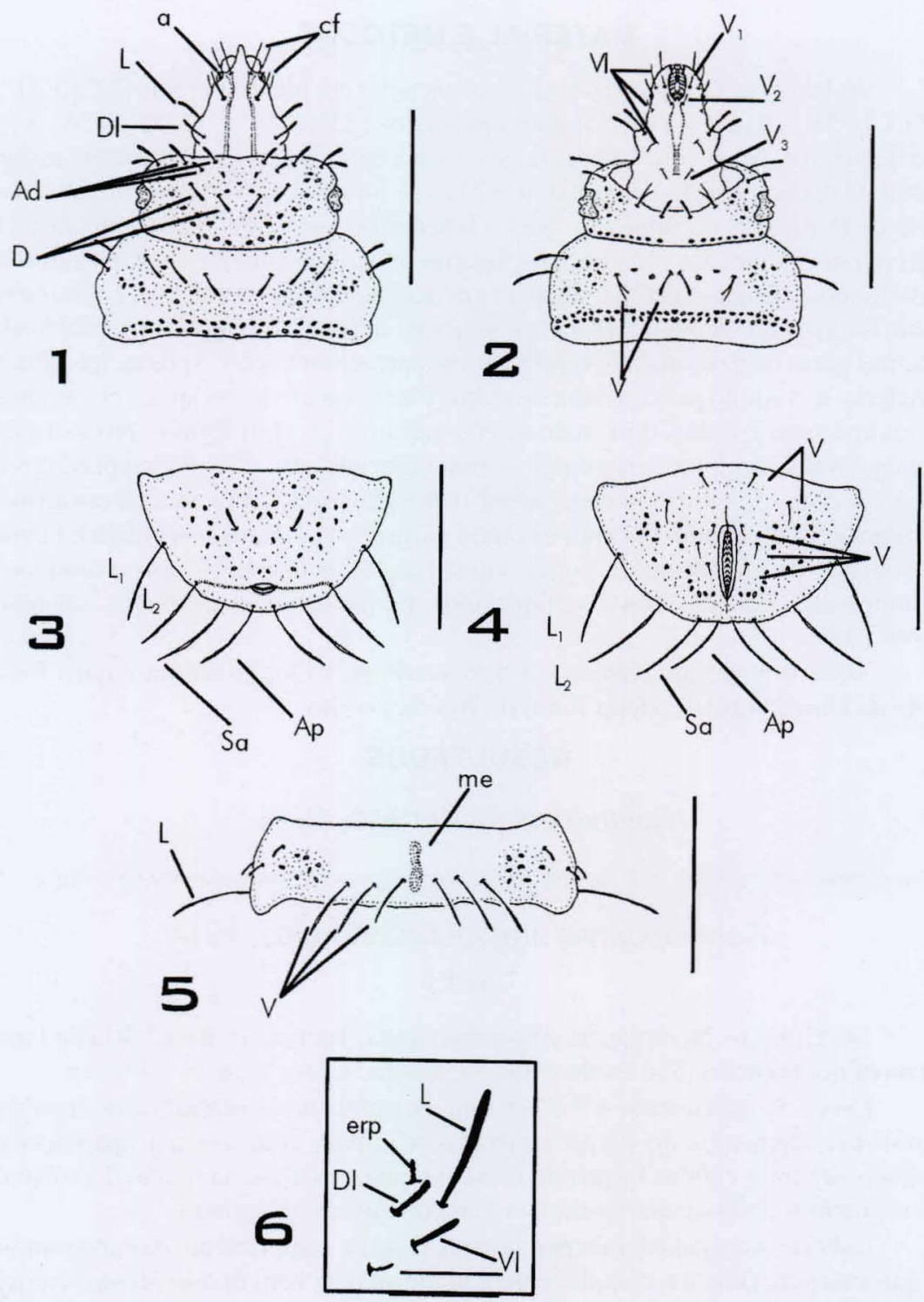

Figs 1-6. Cosmariomyia argyrosticta. (1) Cabeça, primeiro e segundo segmentos torácicos (vista dorsal); (2) cabeça, primeiro e segundo segmentos torácicos (vista ventral); (3) oitavo segmento abdominal (vista dorsal); (4) oitavo segmento abdominal (vista ventral); (5) sexto segmento abdominal (vista ventral); (6) esquema das cerdas da região lateral dos segmentos abdominais. (a) Antena, (Ad) cerdas anterodorsais, (Ap) cerdas apicais, (cf) cerdas clipeofrontais, (D) cerdas dorsais; (DI) cerdas dorsolaterais, (erp) espiráculo respiratório pupal, (L) cerdas laterais, (me) mancha esternal, (Sp) cerdas subapicais, (V) cerdas ventrais, (VI) cerdas ventrolaterais. Barra de escala $=1 \mathrm{~mm}$. 
Primeiro segmento com duas fileiras de cerdas: dois pares de cerdas anterodorsais e três pares de cerdas dorsais (Fig. 1); dois pares de cerdas ventrais sendo o par mais externo bifurcado (Fig. 2). Segundo e terceiro segmentos com uma fileira de cerdas com três pares de cerdas dorsais, dois pares de cerdas ventrais sendo o par mais externo bifurcado, um par de cerdas laterais e um par de cerdas ventrolaterais.

Abdômen. Segmentos 1-7 com a mesma forma, uma fileira de três pares de cerdas dorsais, sendo o par mais externo menor do que os outros e três pares de cerdas ventrais (Fig. 5) sendo o par mais externo maior do que os outros, quatro pares de cerdas laterais abdominais (um par dorsolateral, um par lateral, dois pares ventrolaterais) (Fig. 6). Mancha esternal do sexto segmento com uma característica mancha elíptica (Fig. 5). Segmento 8 com um par de cerdas dorsocentrais (Fig. 3); cinco pares de cerdas ventrais; dois pares de cerdas laterais; um par de cerdas subapicais; um par de cerdas apicais menor que as anteriores (Figs 3 e 4).

Pupário. Com as mesmas características da larva.

Material examinado. BRASIL, Rio de Janeiro: Iguaba Grande, 26.VII.1998 e 01.VIII.1998, R. de Xerez, D.T. Cabral e D.T. de Xerez leg., 10 pupários, 41 exemplares (26 machos e 15 fêmeas) (emergências: 15-17.VIII.1998, 19.VIII.1998, 20.VIII.1998, 22-24.VIII.1998, 24.VIII.1998, 28.VIII.1998, 04.IX.1998, 05-08.IX. 1998, 08.IX.1998, 09.IX.1998, 09.IX.1998, 18.IX.1998, 21.IX.1998, 28.IX.1998, 30.IX.1998, 09.X.1998, 10.XII.1998), e 4 larvas (último instar).

\section{Dactylodeictes Kertész, 1914}

Dactylodeictes Kertész, 1914: 544. Espécie tipo, Dactylodeictes amazonicus Kertész (des. orig.).

\section{Dactylodeictes lopesi Lindner, 1964}

Figs $7-12$

Distribuição. Neotropical: Brasil: Rio de Janeiro (nova ocorrência) e Santa Catarina; México (Morelos, Nayarit, San Luis Potosi e Veracruz); Panamá e Trinidad,

Pupário. Comprimento 5,1 mm, achatado dorsoventralmente, margens laterais dos segmentos do corpo fortemente arqueadas. Cutícula com a aparência usual de mosaico. Padrão cromático marrom-amarelado.

Cabeça. Não achatada dorsoventralmente (Fig. 7); comprimento aproximadamente do mesmo tamanho da largura (Figs 7 e 8); complexo mandíbulo-maxilar bem desenvolvido, labro triangular. Antena pequena, surgindo na parte anterior da cabeça. Quetotaxia: dois pares de cerdas clipeofrontais; um par de cerdas dorsolaterais inseridas acima dos olhos; um par de cerdas laterais inseridas abaixo dos olhos; três pares de cerdas ventrolaterais e três pares de cerdas ventrais em forma de espinho (Figs 7 e 8).

Tórax. Primeiro segmento menor que os outros; espiráculo com dois pares de cerdas à frente, um par dorsolateral e um par ventrolateral (Fig. 7). Primeiro segmento com duas fileiras de cerdas: três pares de cerdas anterodorsais e dois pares de cerdas dorsais (Fig. 7); dois pares de cerdas ventrais sendo o par mais externo 

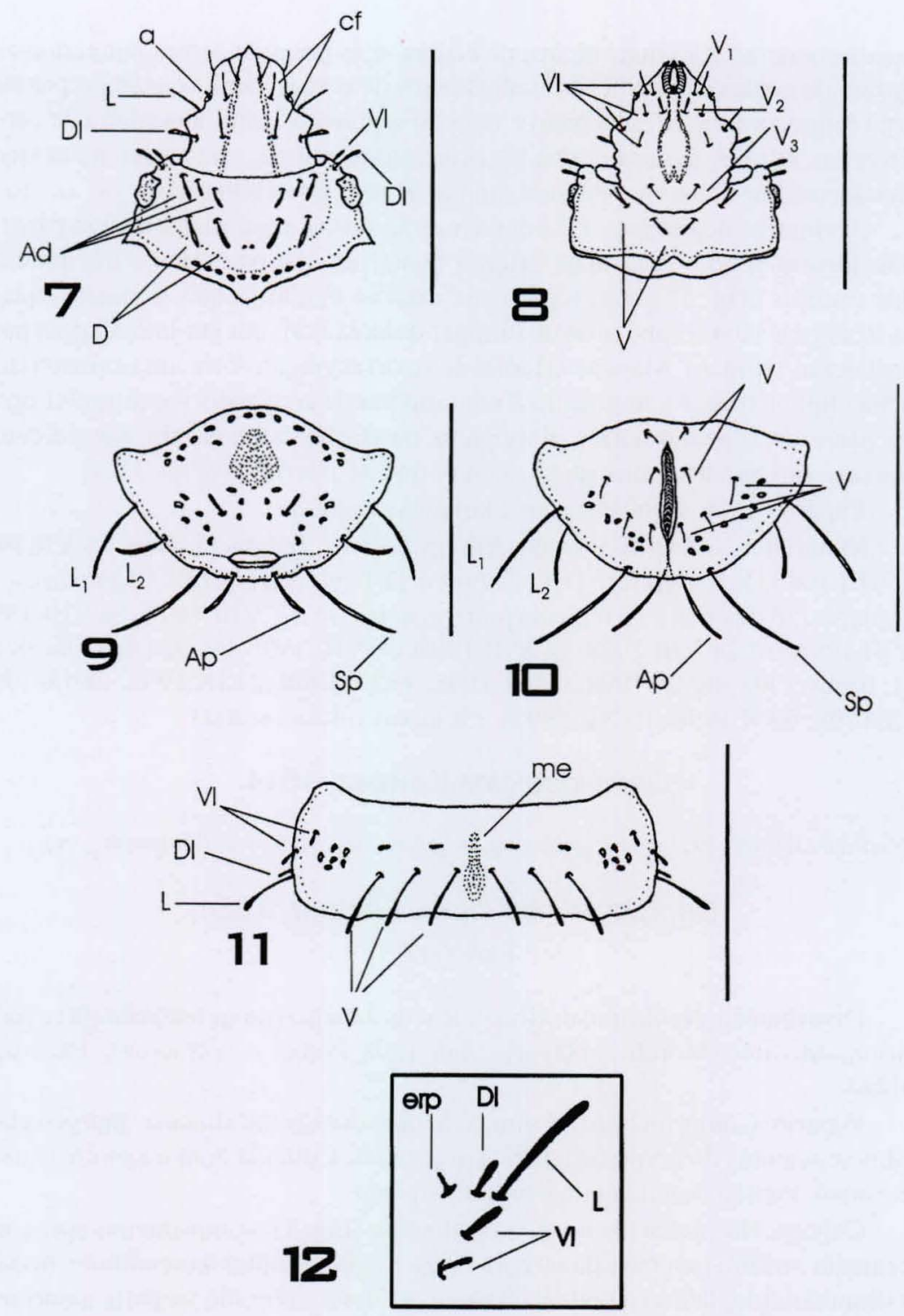

Figs 7-12. Dactylodeictes lopesi, pupário. (7) Cabeça e primeiro segmento torácico (vista dorsal); (8) Cabeça e primeiro segmento torácico (vista ventral); (9) oitavo segmento abdominal (vista dorsal); (10) oitavo segmento abdominal (vista ventral); (11) sexto segmento abdominal (vista ventral); (12) esquema das cerdas da região lateral dos segmentos abdominais. (a) Antena, (Ad) cerdas anterodorsais, (Ap) cerdas apicais, (cf) cerdas clipeofrontais, (D) cerdas dorsais, (DI) cerdas dorsolaterais, (erp) espiráculo respiratório pupal, (L) cerdas laterais, (me) mancha esternal, (Sp) cerdas subapicais, (V) cerdas ventrais, (VI) cerdas ventrolaterais. Barra de escala $=1 \mathrm{~mm}$. 
bifurcado (Fig. 8). Segundo e terceiro segmentos com uma fileira de cerdas com três pares de cerdas dorsais; dois pares de cerdas ventrais sendo o par mais externo bifurcado; um par de cerdas laterais e um par de cerdas ventrolaterais.

Abdômen: Segmentos 1-7 com a mesma forma, com uma fileira de três pares de cerdas dorsais sendo o par mais externo menor que o par mais interno e o mediano, três pares de cerdas ventrais sendo o par mais externo maior do que os outros (Fig. 11), quatro pares de cerdas laterais abdominais (um par dorsolateral, um par lateral, dois pares ventrolaterais) (Fig. 12); linha ventromedial do sexto segmento com uma característica mancha elíptica com a região central estreitada (Fig. 11); segmento 8 arredondado, com um par de cerdas dorsocentrais (Fig. 9); cinco pares de cerdas ventrais (Fig. 10); dois pares de cerdas laterais, sendo o segundo par voltado para cima, um par subapical e um par apical; no lado dorsal apresenta uma elevação acima das cerdas dorsocentrais (Figs 9 e 10).

Material examinado. BraSIL, Rio de Janeiro: Tamoios, Barra de São João, Rodovia RJ-106, Km 127, 25.VII.1998, R. de Xerez leg., 1 pupário, 1 macho (emergência: 14.VIII.1998).

\section{DISCUSSÃO}

Cosmarionyyia argyrosticta pode ser identificada pela seguinte combinação de caracteres: cabeça com o comprimento maior do que a largura, achatada dorsoventralmente, primeiro segmento torácico com dois pares de cerdas anterodorsais, três pares de cerdas dorsais e dois pares de cerdas ventrais sendo o par mais externo bifurcado; segundo e terceiro segmentos torácicos com três pares de cerdas dorsais, dois pares de cerdas ventrais sendo o par mais externo bifurcado, um par de cerdas laterais e um par de cerdas ventrolaterais; abdômen com três pares de cerdas dorsais, sendo o mais externo maior do que os outros, três pares de cerdas ventrais sendo o mais externo maior do que os outros; mancha esternal do sexto segmento elíptica. Dactylodeictes lopesi difere de C. argyrosticta por apresentar cabeça com o comprimento aproximadamente do mesmo tamanho que a largura e não achatada dorsoventralmente; no primeiro segmento torácico apresenta três pares de cerdas anterodorsais e dois pares de cerdas dorsais; mancha esternal do sexto segmento elíptica com as extremidades anterior e posterior afinadas; face dorsal do oitavo segmento com uma pequena elevação acima das cerdas dorsocentrais.

Um aspecto observado no pupário destas duas espécies, assim como em pupários das duas outras espécies estudadas pelos autores, foi o aparecimento de uma linha transversal entre o primeiro e segundo segmento torácicos e de uma linha dorso-mediana a partir do segundo segmento torácico atingindo até o terceiro segmento torácico e às vezes até o primeiro segmento abdominal. ROZKOŠNÝ \& KOVAC (1991) descreveram o pupário de Camptopteromyia fractipennis (Pachygastrinae) e citaram a presença de um pequeno espiráculo respiratório pupal em forma de bastão nas laterais dos segmentos abdominais 1-5, próximo à cerda lateral. Os mesmos autores (RoZKOŠNÝ \& KOVAC 1998a), ao descreverem o pupário de Pachygaster piriventris, citaram a presença dos mesmos espiráculos com a mesma forma numa posição dorsolateral entre as cerdas laterais. Ainda os mesmos autores (ROZKOŠNÝ \& KOVAC 1998b) comentaram que o período pupal pode ser detectado por algumas característi-

Revta bras. Zool. 19 (3): 747 - 755, 2002 
cas, dentre as quais a presença de espiráculos respiratórios pupais inconspícuos, pequenos e em forma de bastão, presentes na região dorsolateral dos segmentos abdominais 1-6 (Beridinae e Pachygastrinae) ou 2-5 (Clitellariinae). As larvas de $C$. argyrosticta e $D$. lopesi também apresentaram estes espiráculos numa posição dorsolateral nos segmentos abdominais 1-6 porém C. argyrosticta apresentou este espiráculo entre as cerdas dorsolateral e lateral formando um pequeno triângulo com as inserções das cerdas (Fig. 6) e D. lopesi apresentou o espiráculo formando uma linha reta com as cerdas dorsolateral e lateral (Fig. 12) Cosmariomyia aurata também apresentou estes espiráculos como em $C$. argyrosticta e $V$. schnusei os apresentou na mesma disposição, porém nos segmentos abdominais 1-7.

Um outro aspecto observado em $C$. argyrosticta foi o aparecimento destes espiráculos respiratórios pupais, pois, como havia sido coletado um grande número de larvas, foi possível fazer a observação de que, à medida que ocorria o desenvolvimento da pupa, mais visível se tornavam estes espiráculos, acreditando desta forma que o desenvolvimento destes seja gradual.

As manchas esternais apresentam-se com a forma elíptica, porém com variações entre as quatro espécies até agora estudadas. Cosmariomyia argyrosticta apresenta uma forma elíptica sem estreitamentos em toda a sua extensão. Dactylodeictes lopesi apresenta esta mancha com um ligeiro estreitamento na sua região central e as extremidades arredondadas. Em $C$. aurata a mancha esternal é afinada nas extremidades e em $V$. schnusei esta mancha é muito semelhante a $D$. lopesi, porém mais fina e a região central mais afinada.

KRAFT \& COOK (1961), MCFADDEN (1967), JAMES (1967a), KRIVOSHEINA (1977), RoZKoŠnÝ \& KovaC (1998a), Pujol-LuZ \& XereZ (1999), XereZ \& PUJOL-LUZ (2001) citaram que normalmente as larvas de Pachygastrinae estão associadas a árvores ou galhos de árvores caídos e em decomposição, onde são encontradas sob a casca. O grau de decomposição da árvore pode influenciar na presença ou não das larvas. Nas coletas, quando procurava-se sob a casca de árvores recém-caídas ou num adiantado estado de decomposição, não era encontrada nenhuma larva. Acreditou-se que este fato se relaciona com o hábito alimentar das larvas, que, segundo KRAFT \& COOK (1961), MCFADDEN (1967), TESKEY (1976), é seiva fermentada, esporos de fungos ou microorganismos produtores de decomposição, provavelmente bactérias decompositoras. As larvas de C. argyrosticta foram coletadas em troncos que apresentavam um certo grau de umidade e uma grande quantidade de fungos e restos de material em decomposição, o que corrobora a afirmativa dos autores supracitados. As larvas de $D$. lopesi foram coletadas num tronco parcialmente descascado e exposto ao sol, o que explicaria a pouca quantidade de larvas presentes. Em troncos com formigas não foram coletadas larvas.

AGRADECIMENTOS. Aos meus filhos Daniel Tavares Cabral e Diogo Tavares de Xerez pela ajuda na coleta das larvas. Ao Curso de Pós-Graduação em Biologia Animal (UFRRJ) pelas facilidades oferecidas durante a realização da minha tese de Doutorado ( $\mathrm{RdX})$. Ao Conselho Nacional de Desenvolvimento Científico e Tecnológico - CNPq pelo auxílio processo $\mathrm{n}^{\varrho}$ 300265/96-4 (JRPL) e ao Programa Institucional de Bolsas de Iniciação Científica da Universidade Federal Rural do Rio de Janeiro (GGV). 


\section{REFERÊNCIAS BIBLIOGRÁFICAS}

Blanchard, E.E. 1923. Apuntes sobre dos dípteros argentinos. Physis, Buenos Aires, 6: 319-323.

JAMES, M.T. 1965. Contributions to our knowledge of the nearctic Pachygasterinae (Diptera: Stratiomyidae). Ann. Entomol. Soc. Amer., College Park, 58: 902-908.

- 1967a. Bredin-Archbold-Smithsonian Biological Survey of Dominica. 5. Family Stratiomyidae (Diptera). Proc. U.S. Nat. Mus., Washington, DC, 123 (3622): 1-22.

1967b. A preliminary review of the Argentine genera and species of Stratiomyidae (Diptera).

Part 2. Pachygasterinae. Acta Zool. Lilloana, Tucuman, 21: 95-121.

- 1981. Stratiomyidae, pp 497-511. In McAlpine, J. F.; B. V. Peterson; G. E. Shewell; H. J.

Teskey; J. R. Vockeroth and D. M. Wood (eds.). Manual of Neartic Diptera. Volume I. Agriculture Canada, Ottawa. I-VI; 1-674.

James, M.T.; M.W. McFadden \& N.E. Woodley. 1980. The Pachygastrinae (Diptera, Stratiomyidae) of Middle America. Melanderia, Pullman, 34: 1-36.

Kertész, K. 1914. Vorarbeiten zu einer Monographie der Notacanthen. XXIII-XXXV. Ann. Mus. Nat. Hungarici, Budapest, 12: 449-557.

KRAFT, K.J. \& E.F. CooK. 1961. A revision of the Pachigasterinae (Diptera, Stratiomyidae) of America North of Mexico. Misc. Publ. Entomol. Soc. Amer., Washington, DC, 3 (1): 1-24.

Krivosheina, N.P. 1977. Xylophilous larvae of soldier flies of the subfamily Pachygasterinae (Diptera, Stratiomyidae). Entomol. Rev., Washington, DC, 56 (4): 113-122.

LindNeR, E. 1964. Beitrag zur Kenntnis der neotroposchen Pachygasterinae (Stratiomyidae, Dipt.). Stuttg. Beitr. Naturkd., Stuttgart, 129: 1-22.

McFadden, M.W. 1967. Soldier fly larvae in America North of Mexico. Proc. U.S. Nat. Mus., Washington, DC, 121 (3569): 1-72.

Pujol-LuZ, J.R. \& R. DE XEREz. 1999. The larva of Chalcidomorphina aurata Enderlein, 1914 (Diptera: Stratiomyidae) from "Ilha da Marambaia", Rio de Janeiro, Brazil. Proc. Entomol. Soc. Wash. 101: 295-299.

Rozkošný, R. \& D. KovaK. 1991. First description of the male and the larva of Camptopteromyia fractipennisde Meijere from Malaysia (Diptera: Stratiomyidae). Entomol. Scand., Stenstrup, 22 (3): 297-304.

RozkoŠnÝ, R. \& D. KovAK. 1998a. A new species of Pachygaster (Diptera: Stratiomyidae, Pachygasterinae), from West Malaysia and Thailand. Studia Dipterol., Halle (Saale), 5 (1): 3-12.

- 1998b. Descriptions of bamboo-inhabiting larvae and puparia of Oriental soldier flies Ptecticus brunettii and P. flavi femoratus (Diptera: Stratiomyidae: Sarginae) with observations on their biology. Eur. Jour. Entomol, Branisovska, 95: 65-86.

Teskey, H.J. 1976. Diptera larvae associated with trees in North America. Mem. Entomol. Soc. Can., Ottawa, 100: 1-53.

Woodley, N.E. 1989. 33. Family Stratiomyidae, p. 301-320. In: N.L. Evenhuis (Ed.). Catalog of the Diptera of the Australasian and Oceanian Regions. Leiden, Bishop Museum Press and E.J. Brill, $1155 \mathrm{p}$.

- 1995. The genera of Beridinae (Diptera, Stratiomyidae). Mem. Entomol. Soc. Wash. 16: 1-231. 1-473.

Xerez, R. DE \& J.R. Pujol-Luz. 2001. Description of the larva of Vittiger schnusei Kertész, 1909 (Diptera, Stratiomyidae) from Ilha da Marambaia, Rio de Janeiro, Brasil. Studia Dipterol., Halle (Saale), 8 (1): 337-341.

Recebido em 13.III.2002; aceito em 24.VII.2002. 